\title{
Dual-trap optical tweezers with real-time force clamp control
}

\section{Wallin, Anders}

2011-08-03

Wallin , A , Ojala , H V , Ziedaite , G \& Haeggström , E 2011 , ' Dual-trap optical tweezers with real-time force clamp control ' Review of Scientific Instruments, vol. 82 , no. 8 , pp. 083102 . DOI: 10.1063/1.3615309

http://hdl.handle.net/10138/27789

https://doi.org/10.1063/1.3615309

Downloaded from Helda, University of Helsinki institutional repository.

This is an electronic reprint of the original article.

This reprint may differ from the original in pagination and typographic detail.

Please cite the original version. 


\title{
Dual-trap optical tweezers with real-time force clamp control
}

\author{
Anders E. Wallin*, Heikki Ojala, Gabija Ziedaite, and Edward Hæggström
}

Department of Physics, University of Helsinki, P.O.Box 64, FIN-00014 Helsinki, FINLAND

\begin{abstract}
Single molecule force clamp experiments are widely used to investigate how enzymes, molecular motors, and other molecular mechanisms work. We developed a dual-trap optical tweezers instrument with real-time ( $200 \mathrm{kHz}$ update rate) force clamp control that can exert $0-100 \mathrm{pN}$ forces on trapped beads. A model for force clamp experiments in the dumbbell-geometry is presented. We observe good agreement between predicted and observed power spectra of bead position and force fluctuations. The model can be used to predict and optimize the dynamics of real-time force clamp optical tweezers instruments. The results from a proof-of-principle experiment in which lambda exonuclease converts a double-stranded DNA tether, held at constant tension, into its singlestranded form, show that the developed instrument is suitable for experiments in single molecule biology.
\end{abstract}

keywords: optical tweezers, feedback control, single molecule biology

*) Electronic mail: anders.wallin@ @elsinki.fi

Copyright (2011) American Institute of Physics. This article may be downloaded for personal use only. Any other use requires prior permission of the author and the American Institute of Physics.

The following article appeared in Review of Scientific Instruments 82, 083102, (2011) and may be found at (http://link.aip.org/link/doi/10.1063/1.3615309). 


\section{Introduction}

Micromechanical experiments in single-molecule science using optical tweezers (OT), magnetic tweezers, or atomic force microscopes (AFMs) typically probe macromolecular interactions and conformations using an elastic probe (a trapped bead or an AFM tip) attached to the sample through a compliant tether ${ }^{1}$. Constant force experiments are preferred because they observe movement of the probe without contributions from length-changes of the tether due to variations in force ${ }^{2,3}$. Constant force experiments have been used for observing e.g. molecular motors ${ }^{4}$ or DNA/RNA hairpin unfolding ${ }^{5}$. Force clamp OT experiments can be performed by controlling one end of the tether using a piezo-stage ${ }^{6}$, modulating the trap laser intensity ${ }^{7}$, by steering the optical trap using acousto-optical deflectors ${ }^{8}$, or by scanning mirrors that create a constant optical gradient field ${ }^{9}$. Particles can also be trapped in the constant-force region of the optical potential ${ }^{10,11}$, where over $50 \mathrm{~nm}$ extension the force is constant to within $5 \%$. Molecular events such as DNA hairpin unfolding or stepping by a molecular motor can occur on timescales ranging from microseconds to milliseconds, exhibit forces up to $50 \mathrm{pN}$, and produce cumulative changes in extension exceeding several micrometers. Consequently there is a need for a high-bandwidth micromechanical forceclamp with sufficient force- and extension-range.

Based on previous work with position clamp control of trapped particles ${ }^{12,13}$, we present a dual-trap OT instrument with real-time force clamp control. Our dumbbell experiment is controlled by a data acquisition card incorporating a field-programmable gate-array (FPGA) with $200 \mathrm{kHz}$ update rate, which maintains constant force in a DNA tether. A model that accounts for the elasticity of both traps and the tether and for the thermal motion of the beads agrees well with the observed position and force power spectral densities. The model can thus be used to predict the dynamics of force clamp experiments when experimental parameters such as tether length/tension, or feedback gains are varied. A proof-of-principle single molecule experiment was performed by force-clamping a double-stranded DNA molecule in the presence of lambda exonuclease, which digests one strand of the molecule and gradually converts the tether to single-stranded DNA. The change in length of the force-clamped tether during the enzymatic digestion reaction was monitored.

\section{Theory}

A plot of the measured power spectral density (PSD) of bead position fluctuations is commonly used for calibrating optical tweezers by comparing it to the predicted Lorentzian PSD ${ }^{14}$. Here we derive expressions for the PSDs of bead and trap position fluctuation during real-time forceclamping.

A typical 'dumbbell' construct, Fig. 1, used in high-resolution OT experiments consists of two optically levitated beads connected by an elastic DNA tether. Ignoring the inertia of the beads and assuming that over small changes in extension the tether behaves as a Hookean spring, the equation of motion for the system is 15

$$
-\boldsymbol{\mu}^{-1} \dot{\mathbf{x}}(t)-\mathbf{\kappa} \mathbf{x}(t)+\mathbf{k} \mathbf{x}_{\mathbf{T}}(t)=\mathbf{F}(t)
$$

where $\mathbf{x}(t), \quad \mathbf{x}_{T}(t)$, and $\mathbf{F}(t)$ denote deviations from an equilibrium position/force, and $\mathbf{x}(t)=\left[\begin{array}{ll}x_{1}(t) & x_{2}(t)\end{array}\right]^{T}$ is the position of the beads, $\mathbf{x}_{T}(t)=\left[\begin{array}{lll}x_{T 1}(t) & x_{T 2}(t)\end{array}\right]^{T}$ is the position of the optical traps, and $\mathbf{F}(t)=\left[\begin{array}{lll}F_{1}(t) & F_{2}(t)\end{array}\right]^{T}$ is a random force due to thermal motion. The mobility matrix $\boldsymbol{\mu}$ and stiffness matrices $\mathbf{K}$ and $\mathbf{k}$ are 


$$
\boldsymbol{\mu}^{-1}=\left(\begin{array}{cc}
\gamma_{1} & 0 \\
0 & \gamma_{2}
\end{array}\right), \mathbf{\kappa}=\left(\begin{array}{cc}
k_{1}+k_{D N A} & -k_{D N A} \\
-k_{D N A} & k_{2}+k_{D N A}
\end{array}\right), \mathbf{k}=\left(\begin{array}{cc}
k_{1} & 0 \\
0 & k_{2}
\end{array}\right)
$$

where $\gamma_{1,2}$ is the hydrodynamic drag-coefficient, $k_{1,2}$ denotes trap stiffness, and $k_{D N A}$ is the local spring-constant of the tether. In Eq. (2) we have omitted hydrodynamic interaction between the beads, since we expect it to be negligible in our experiments using a DNA tether that is long (16 $\mu \mathrm{m})$ compared to the bead radius. In our experiment the left trap is held stationary $\left(x_{T 1}(t)=0\right)$ while the right trap is steered using a proportional-integral (PI) controller. The error signal for the PI-controller is given by the difference in measured force $k_{1} x_{1}(t)$ in the stationary trap compared to a set-point force $F_{\text {set }}$, the desired tether tension. Ignoring constant terms, the position fluctuation of the steerable trap is

$$
\begin{aligned}
& x_{T 2}(t)=\frac{G_{P}}{k_{1}} F_{e r r}(t-\tau) \\
& +\frac{G_{I}}{k_{1}}\left[F_{e r r}(t-\tau)+F_{e r r}(t-\tau-\Delta)+F_{e r r}(t-\tau-2 \Delta)+\ldots\right]
\end{aligned}
$$

where $F_{\text {err }}=F_{\text {set }}-k_{1} x_{1}(t)$ is the force error and $G_{P}$ and $G_{I}$ denote the proportional and integral feedback gains, respectively. Equation (3) takes into account the loop delay time $\tau$ i.e. the time it takes for the controller to measure $x_{1}$ and react by steering $x_{T 2}$, as well as the sampling period $\Delta$, i.e. the data collection interval. For our instrument $\tau \approx 19 \mu \mathrm{s}$ and $\Delta=5 \mu \mathrm{s}{ }^{12}$ Fourier transforming Eq. (3) gives

$$
\begin{aligned}
& \tilde{x}_{T 2}(\omega)=G_{P} \tilde{x}_{1}(\omega) \exp (-i \omega \tau) \\
& +G_{I} \tilde{x}_{1}(\omega) \exp (-i \omega \tau) \frac{1}{1-\exp (-i \omega \Delta)},
\end{aligned}
$$

which can be inserted into Eq. (1) to yield the equations of motions

$$
\left\{\begin{array}{c}
T_{1} \tilde{x}_{1}(\omega)+k_{D N A} \tilde{x}_{2}(w)=\tilde{F}_{1}(w) \\
T_{2} \tilde{x}_{2}(\omega)+k_{D N A} \tilde{x}_{1}(\omega)-k_{F} \tilde{x}_{1}(\omega)=\tilde{F}_{2}(\omega)
\end{array}\right.
$$

where, for compactness, we have used the symbols:

$$
\begin{aligned}
& T_{j} \equiv-i \omega \gamma_{j}-k_{j}-k_{D N A}, \quad j=1,2 \\
& k_{F} \equiv k_{2} \exp (-i \omega \tau)\left(G_{P}+\frac{G_{I}}{1-\exp (-i \omega \Delta)}\right) .
\end{aligned}
$$

Solving for the Fourier transform of the bead fluctuations $x_{1}(t)$ and $x_{2}(t)$ gives

$$
\begin{aligned}
& \tilde{x}_{1}(\omega)=\frac{T_{2} \tilde{F}_{1}(\omega)-k_{D N A} \tilde{F}_{2}(\omega)}{T_{1} T_{2}-k_{D N A}^{2}+k_{D N A} k_{F}}, \\
& \tilde{x}_{2}(\omega)=\frac{T_{1} \tilde{F}_{2}(\omega)-\left(k_{D N A}-k_{F}\right) \tilde{F}_{1}(\omega)}{T_{1} T_{2}-k_{D N A}^{2}+k_{D N A} k_{F}}
\end{aligned}
$$


These expressions can be used to find the PSDs of the bead positions $\left|\tilde{x}_{1}(\omega)\right|^{2}$ and $\left|\tilde{x}_{2}(\omega)\right|^{2}$, the force in the stationary trap $\left|k_{1} \tilde{x}_{1}(\omega)\right|^{2}$, the steerable trap position $\left|\tilde{x}_{T 2}(\omega)\right|^{2}=\left|k_{F} k_{2}^{-1} \tilde{x}_{1}(\omega)\right|^{2}$, as well as the PSD of tether extension fluctuation $\left|\tilde{x}_{1}(\omega)-\tilde{x}_{2}(\omega)\right|^{2}$.

\section{Dual-trap optical tweezers with real-time feedback control}

Our dual-trap real-time optical force-clamp instrument is based on earlier work with position-clamp control $^{12,13}$. A schematic overview of the instrument is shown in Fig. 2. A 4 W $1064 \mathrm{~nm} \mathrm{CW-laser}$ (Coherent Compass) is first collimated (L1:L2), then passed through a Faraday isolator (FI, Linos), and split (PBS1) into a stationary and steerable trap with orthogonal polarizations. The steerable trap passes through acousto-optic deflectors (AOD X/Y, NEOS-Technologies) while the stationary trap can be adjusted manually with gimball-mirrors $(\mathrm{M})$. The trapping beams are combined with another polarizing beam-splitter (PBS2) and steered with telescopes (L3:L4) and (L5:L6) via a dichroic mirror (D2, Chroma) into an inverted microscope (Nikon TE-2000) so that the plane between the AODs (dashed line) is imaged onto the back-focal-plane of the microscope objective (Nikon 100x TIRF oil-immersion, N.A. 1.49).

Position detection of trapped particles is performed with dual detection beams at $785 \mathrm{~nm}$ and $830 \mathrm{~nm}$. The light from a temperature stabilized diode-laser (HL7851G, Hitachi, and DL5032-001, Thorlabs) is passed through a Faraday isolator (FI) and coupled to a polarization-maintaining single-mode fiber (SMF) in order to purify the spatial laser mode and to stabilize pointing. Halfwave plates before (HWP2/4) and after the isolator (HWP3/5) adjust the overall intensity and align the polarization to the polarization-maintaining axis of the single mode-fiber, respectively. The position of the detection lasers in the sample plane is adjustable using gimball-mirrors in the backfocal-plane. The orthogonally polarized detection beams are combined at PBS3, expanded with L7:L8 and combined with the trapping beams at dichroic D1. A high numerical aperture condenser (COND, Nikon HNA-OIL, N.A. 1.4) collects detection light which is deflected towards $10 \times 10 \mathrm{~mm}^{2}$ duolateral position-sensitive detectors (PD1/2, SiTek, S2-0171) using dichroic D3. A short-pass filter F1 rejects trapping light at $1064 \mathrm{~nm}$, while a polarizing beam-splitter (PBS4) and laser-line filters centered at $785 \mathrm{~nm}$ and $830 \mathrm{~nm}$ (F2 and F3, Semrock) prevent cross-talk between the detectors. A Galilean telescope (L9:L10) collimates the transmitted light, while focusing lenses (L11 and L12) image the condenser back-focal-plane onto the detector surface. In experiments where the dumbbell extension changes less than $\sim 500 \mathrm{~nm}$ one detection laser monitors the bead in the stationary trap while the other detection laser is positioned so that it monitors the steerable bead. In experiments where the extension change is large, the steerable bead can be monitored in a $\sim 500 \mathrm{~nm}$ window, outside of which the steerable bead position must be inferred from the position of the steerable trap, the steerable trap stiffness, and the tether tension. An LED provides bright-field illumination for a CCD-camera. Short pass filters F4 and F5 attenuate the trapping and detection light to prevent saturation of the camera. A tube-lens (TL) internal to the microscope allows switching between 100x and 150x magnification. The sample is positioned on two stacked stages: a piezo-motor stage (Physik Instrumente, M-686) with $25 \times 25 \mathrm{~mm}^{2}$ travel range and $100 \mathrm{~nm}$ resolution provides coarse sample positioning, while a piezoelectric stage (Physik Instrumente, P$517.3 \mathrm{CD}$ ) with $100 \times 100 \times 30 \mu \mathrm{m}^{3}$ range provides nanometer resolution positioning.

Our instrument is controlled by a personal computer running LabVIEW 2009 software. Figure 3 shows a schematic overview of the control electronics. Data collection, real-time control, and trap steering is performed with a data acquisition card incorporating a Field Programmable Gate Array (FPGA, PCI-7833R, National Instruments). The FPGA-card allows control algorithms to run in real-time with up to $200 \mathrm{kHz}$ update rate, independently of the host operating system and other 
computer peripherals. To match the detection signals to the $\pm 10 \mathrm{~V}$ input of the analog to digital converter (ADC) we use variable-gain instrumentation amplifiers (INA111, Burr-Brown) which combine and amplify the four pre-amplified voltage signals available from the PDs. Each PD also provides a sum-signal which is not amplified. Analog voltages are low-pass filtered with a $60 \mathrm{kHz}$ fourth-order Butterworth anti-aliasing filter (AAF) and then digitized at $200 \mathrm{kS} / \mathrm{s}$ and 16-bit precision. Based on the measured detector-voltages a feedback algorithm implemented on the FPGA calculates AOD steering-commands every $5 \mu \mathrm{s}$. Two 30-bit digital control words are output to two direct digital synthesizers (DDS, NEOS Technologies, $1 \mathrm{GHz}$ internal clock) which drive the AODs with an RF-signal at a variable frequency between 25 and $45 \mathrm{MHz}$ and an adjustable amplitude set by a digital to analog (DAC) output on the FPGA card. Manual control of trap position can be performed with two hand-wheels that output quadrature pulses to the FPGA. This allows the operator to position the trap before an experiment begins, or to perform a force-extension experiment manually prior to force-clamping. Independent of the feedback-loop both bead and trap position data are logged to disk at a maximal rate of $200 \mathrm{kS} / \mathrm{s}$ for later analysis. In long duration experiments, to reduce disk usage, data can be collected at $200 \mathrm{kS} / \mathrm{s}$, digitally low-pass filtered using a digital 2nd order Butterworth low-pass filter on the FPGA, and saved to disk at a reduced sampling rate. Although the update rate for the feedback control algorithm is $200 \mathrm{kHz}$, the bandwidth for closed loop control is limited by other components in the loop: detection, anti-alias filtering, and steering. The position sensitive detectors have a bandwidth of $>200 \mathrm{kHz}$ at the used wavelengths (785 nm and $830 \mathrm{~nm}$ ) as determined with a LED-wobbler ${ }^{16}$ measurement. In order to suppress aliasing above the Nyquist frequency $(100 \mathrm{kHz})$ the corner frequency of the $4^{\text {th }}$ order antialias filter was set at $60 \mathrm{kHz}$. Finally, the speed of sound in the $\mathrm{TeO}_{2}$ crystals $(660 \mathrm{~m} / \mathrm{s})$ of the AOD limits the steering bandwidth to $\sim 100 \mathrm{kHz}$. The overall bandwidth is thus limited to $\sim 60 \mathrm{kHz}$.

\section{Methods}

Two proof-of-principle experiments were performed to test the real-time force-clamp. In the first experiment a $48 \mathrm{~kb}$ double-stranded (ds) DNA molecule was held force-clamped at $6 \mathrm{pN}$ while the feedback gains were varied to minimize $F_{\text {err }}$. In the second experiment we chose a well described enzyme, bacteriophage lambda exonuclease ${ }^{17-19}$, to test our active feedback control during an enzymatic reaction which alters the tether properties. Exonucleases are integral parts of many genetic recombination and repair systems. Bacteriophage lambda exonuclease processively degrades one strand of double stranded DNA in the 5' to 3' direction ${ }^{18}$, leaving non-hydrolyzed single-stranded (ss) DNA and 5'-mononucleotides. In the presence of exonuclease we observe, at constant force, the enzymatic degradation of the unlabeled strand of our DNA-construct, resulting in a gradual conversion of the tether from double-stranded to single-stranded (ss) form. When DNA is held at constant force below $\sim 6 \mathrm{pN}$ the ss-form is significantly shorter than the ds-form, and thus the enzymatic activity can be observed as a gradual shortening of the tether.

A $48 \mathrm{~kb}$ long DNA construct used in both the experiments was prepared from phage lambda DNA (New England Biolabs) by annealing biotinylated oligonucleotides (Thermo Fischer) at both 5'- and 3 '-ends, essentially as described $i^{20}$. Formation of the dumbbell was performed in a 3-channel sample-chamber (Fig. 4). Two streptavidin coated polystyrene beads $(1.87 \mu \mathrm{m}$ diameter, Kisker Biotech) were trapped in the first lane of the chamber, transported through the second lane containing DNA at $3.5 \mathrm{pM}$ concentration, where a tether was formed, before entering the third lane for measurement. The first and second lane contained TEW buffer $(20 \mathrm{mM}$ Tris $\mathrm{pH} 8,1 \mathrm{mM}$ EDTA, $150 \mathrm{mM} \mathrm{NaCl}, 0.05 \mathrm{mg} / \mathrm{ml} \mathrm{BSA}$, and $0.1 \%$ Tween20). In the first experiment the third lane of the chamber contained the same TEW buffer but in the second experiment the third lane contained lambda exonuclease $(125 \mathrm{U} / \mathrm{ml})$ in $1 \mathrm{x}$ exonuclease reaction buffer (both from New England Biolabs). Prior to experiments the whole sample-chamber was blocked with TEW buffer 
containing $5 \mathrm{mg} / \mathrm{ml}$ BSA. A syringe pump with $1 \mathrm{ml}$ syringes was used to introduce beads, DNA, and buffer to the chamber at a rate of $1 \mu \mathrm{l} / \mathrm{min}$. For the first experiment the flow was stopped during the data collection but in the exonuclease experiments the flow was on during the measurement. When a tether was found a force-extension curve was collected in order to distinguish single DNA tethers from multiple tethers. In the first experiment single tethers were then force-clamped at $6 \mathrm{pN}$ while the gains of the PI-controller were varied. Time-series of $2 \mathrm{~s}$ duration were collected for each gain setting. In the second experiment the tether was force-clamped at $3.4 \mathrm{pN}$ and data was collected for $20 \mathrm{~min}$, or until the tether broke.

\section{Results}

In the first experiment a force extension measurement was performed in the third lane of the chamber. The tether was then pulled close to the set-point tension and the feedback control was activated. Figure 5 shows time series data of force and trap position, first without feedback control (red data, $t=0-2 \mathrm{~s}$ ), and then with the feedback control activated, using different $G_{P}$ and $G_{I}$ $(t=2-10 \mathrm{~s})$. A reduction in the force-fluctuation is observed with increasing proportional gain (Fig. 5A). The standard deviation in force was reduced from $0.8 \mathrm{pN}$ to $0.5 \mathrm{pN}$ when feedback was active $\left(G_{P}=55\right.$ and $G_{I}=0.3$ ). As seen from Fig. 5 and Fig. 6 the reduction in tension fluctuation was most dramatic below $100 \mathrm{~Hz}$ where the standard deviation decreased tenfold from $0.2 \mathrm{pN}$ without feedback to $0.02 \mathrm{pN}$ with feedback active. This reduction in tether tension fluctuation is achieved only through an increased trap motion (Fig. 5B). The standard deviation in trap position $x_{T 2}$ with $G_{P}=55$ and $G_{I}=0.3$ was $180 \mathrm{~nm}(20 \mathrm{~nm}$ in $0-100 \mathrm{~Hz})$. In the first experiment data recording and beam steering was performed at the maximum bandwidth of the instrument $(200 \mathrm{kS} / \mathrm{s})$.

Figure 6 shows PSD data from the first experiment. Increasing the integral gain $G_{I}$ reduces the low frequency force fluctuations while increasing the proportional gain $G_{P}$ reduces force fluctuations across all frequencies. The experimental data reproduces the predicted asymptotic frequency dependence of $f^{2}$ and $f^{-2}$ at low and high frequencies, respectively. Figures 6B, C, and D show how fluctuations in steerable bead position, trap position, and tether extension increase at every frequency for all $G_{P}$ and $G_{I}$ values tried. The predicted PSDs from Eq. (7), shown as black lines, agree with the measured data. The predicted PSDs were plotted on top of experimental data without fitting by using a local DNA-stiffness $\left(k_{D N A}=9 \mathrm{pN} / \mu \mathrm{m}\right)$ from a force-extension measurement, the calibrated trap stiffness $\left(k_{1}=150 \mathrm{pN} / \mu \mathrm{m}\right.$ and $\left.k_{2}=380 \mathrm{pN} / \mu \mathrm{m}\right)$, and the known feedback gains $G_{P}$ and $G_{I}$.

In the exonuclease experiment the set-point force was chosen as $F_{\text {set }}=3.4 \mathrm{pN}$. In this long-duration experiment data was collected at $200 \mathrm{kS} / \mathrm{s}$, digitally low-pass filtered to $2 \mathrm{kHz}$ on the FPGA, and stored to disk at $8 \mathrm{kS} / \mathrm{s}$. The results from a representative experiment are shown in Fig. 7. In this experiment the extension of the tether smoothly decreased from $15.4 \mu \mathrm{m}$ to $7.7 \mu \mathrm{m}$ during $\sim 900 \mathrm{~s}$, before the tether broke. The tether tension was held constant at $3.4 \pm 0.4 \mathrm{pN}(2 \mathrm{kHz}$ bandwidth) during the experiment. To estimate the rate of enzymatic activity the extension data was windowed into $10 \mathrm{~s}$ time intervals with $95 \%$ overlap. First order least-square fits to these windows yielded velocity data which is shown in Fig. 7C, and shown histogrammed in Fig. 7D. The average velocity with which the tether length decreased was $9 \pm 6 \mathrm{~nm} / \mathrm{s}$. This corresponds to a velocity of $30 \mathrm{nt} / \mathrm{s}$ of the lambda exonuclease (assuming a $14 \mu \mathrm{m}$ length change during $48 \mathrm{~kb}$ of translocation ${ }^{20}$ 
), in agreement with previous measurements ${ }^{17}$. Figure 8 shows a frame from a video recording of the lambda exonuclease experiment.

\section{Discussion and Conclusions}

The proposed active real-time optical force-clamp can maintain a constant 0-100 pN tension in a dumbbell-tether while allowing for tether extension changes of several micrometers. The downside of this control is the increased fluctuation in the steerable trap position, and consequently the tether extension. However, our results show that the predicted PSDs agree with experimental data and thus Eq. (7) allows designing real-time force-clamp experiments that balance the trade-off between the conflicting requirements of constant tether tension and low uncertainty in the tether extension. At the highest proportional gains used, $G_{P}=55$, a resonance peak at $\sim 3 \mathrm{kHz}$ is predicted but not visible in the experimental data (Fig. 6). This may be because Eq. (1) does not account for the drag of the DNA tether, which is similar to that of the beads ${ }^{21}$. At all other gains the good agreement between theory and data in Fig. 6 indicates that the longitudinal relaxation time of DNA, which is in the millisecond range at high extension ${ }^{21}$, does not influence the dynamics significantly. We conclude that using Eq. (7) it is possible to predict what effect varying the instrument bandwidth, the loop delay, the set-point force, and the feedback gains will have on the shape of force and position PSDs when force-clamping elastic tethers. Our results using integral gain show that the force error PSD is proportional to $f^{2}$ from low frequencies up to around the trap corner frequency. This suggests that in order to force-clamp with minimum error, instruments should feature feedback control with a bandwidth extending to at least the corner frequency. When trapping stiff constructs at high forces, e.g. when trying to detect short steps of molecular motors ${ }^{22}$, a bandwidth of $\geq 10 \mathrm{kHz}$, typically requiring an FPGA or Digital Signal Processor based controller, is thus required.

The second experiment performed with lambda exonuclease shows that our instrument can track extension-changes over several micrometers at constant tension. In future work the forceclamp will be applied to fast biological events such as RNA/DNA hairpin unfolding experiments, or studying fast strong molecular motors. It may also be possible to determine what bandwidth and feedback gains are sufficient or optimal for a certain force-clamp experiment, or to design novel improved control algorithms.

\section{Acknowledgements}

This work was supported by an Academy of Finland grant (nr 128518) to E.H. H.O. acknowledges support from the Finnish Academy of Science and Letters (Väisälä Foundation). We thank Dr. Roman Tuma for critical reading of the manuscript. 


\section{References}

1. K. C. Neuman and A. Nagy, Nat. Methods 5, 491 (2008).

2. K. Visscher and S. M. Block, Methods Enzymol. 298, 460 (1998).

3. F. Gittes and C. F. Schmidt, Eur. Biophys. J. 27, 75 (1998).

4. D. E. Smith, S. J. Tans, S. B. Smith, S. Grimes, D. L. Anderson and C. Bustamante, Nature 413, 748 (2001).

5. M. T. Woodside, P. C. Anthony, W. M. Behnke-Parks, K. Larizadeh, D. Herschlag and S. M. Block, Science 314, 1001 (2006).

6. G. J. Wuite, R. J. Davenport, A. Rappaport and C. Bustamante, Biophys J 79, 1155 (2000).

7. M. D. Wang, H. Yin, R. Landick, J. Gelles and S. M. Block, Biophysical Journal 72, 1335 (1997).

8. M. J. Lang, C. L. Asbury, J. W. Shaevitz and S. M. Block, Biophys. J. 83, 491 (2002).

9. R. Nambiar, A. Gajraj and J-C Meiners, Biophysical Journal 87, 1972 (2004).

10. W. J. Greenleaf, M. T. Woodside, E. A. Abbondanzieri and S. M. Block, Physical Review Letters 95, 208102 (2005).

11. Y. F. Chen, G. A. Blab and J. C. Meiners, Biophys. J. 96, 4701 (2009).

12. A. E. Wallin, H. Ojala, E. Haeggström and R. Tuma, Applied Physics Letters 92, 224104 (2008).

13. H. Ojala, A. Korsback, A. E. Wallin and E. Haeggstrom, Applied Physics Letters 95, 181104 (2009).

14. K. Berg-Sorensen and H. Flyvbjerg, Review of Scientific Instruments 75, 594 (2004).

15. Jeffrey R. Moffitt, Yann R. Chemla, David Izhaky and Carlos Bustamante, Proc. Natl. Acad. Sci. USA. 103, 9006 (2006).

16. J. Huisstede, B. van Rooije, K. van der Werf, M. Bennink and Subramaniam V, Optics Letters 31, 610 (2006).

17. T. T. Perkins, R. V. Dalal, P. G. Mitsis and S. M. Block, Science 301, 1914 (2003).

18. P. G. Mitsis and J. G. Kwagh, Nucleic Acids Res. 27, 3057 (1999).

19. K. Subramanian, W. Rutvisuttinunt, W. Scott and R. S. Myers, Nucleic Acids Res. 31, 1585 (2003).

20. N. A. Tanner and A. M. Van Oijen, Methods in Molecular Biology 521, 397 (2009).

21. J-C Meiners and S. R. Quake, Phys. Rev. Lett. 84, 5014 (2000).

22. A. E. Wallin, A. Salmi and R. Tuma, Biophysical Journal 93, 795 (2007). 


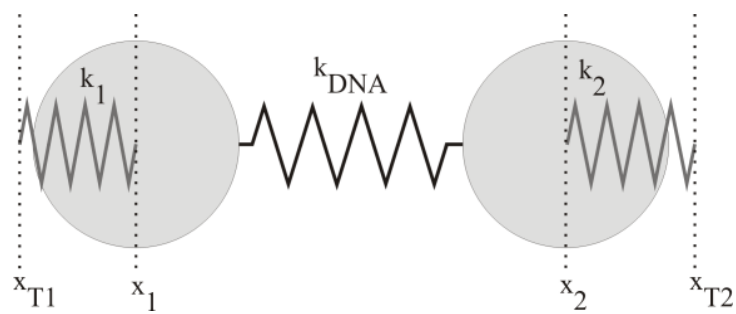

FIG. 1. Geometry and symbols used for describing a dual-trap 'dumbbell' experiment

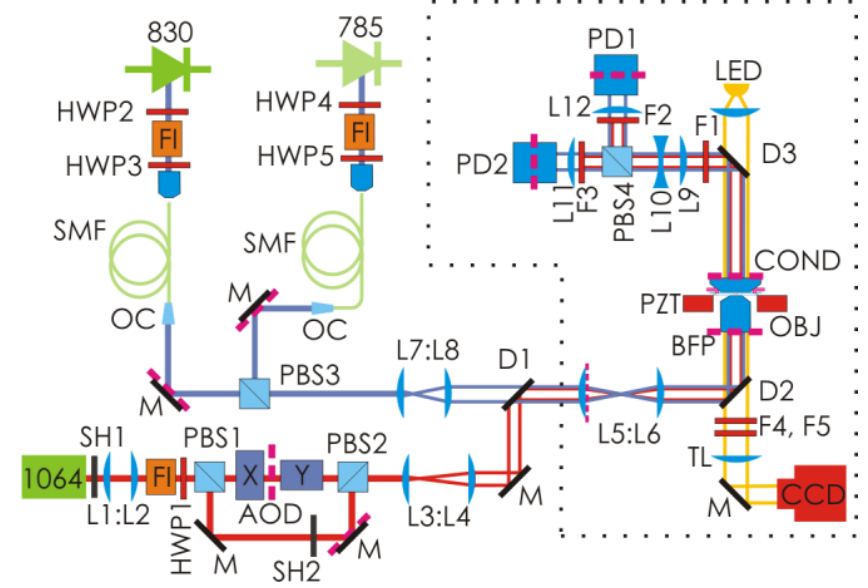

FIG. 2. (Color online) Dual-trap optical tweezers instrument with two detection lasers. Red dashed lines indicate optically conjugate planes. Components inside the dotted line are mounted on/inside the microscope body. Trap laser (1064), shutter (SH), lens (L), half-wave plate (HWP), Faraday isolator (FI), polarizing beam-splitter (PBS), single-mode fiber (SMF), output-coupler (OC), mirror $(\mathrm{M})$, dichroic mirror (D), objective (OBJ), piezo-electric stage (PZT), condenser (COND), filter (F), position-sensitive photodiode (PD), tube-lens (TL). 


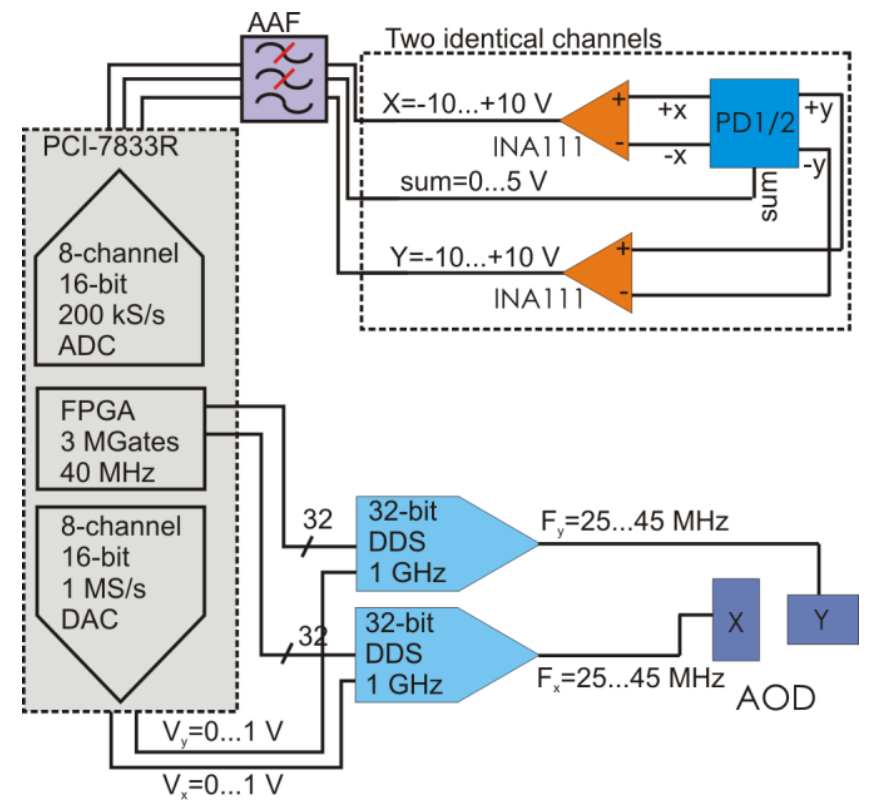

FIG. 3. (Color online) Electrical schematic of real-time force-clamp instrument. Data acquisition card (PCI-7833R) with field-programmable gate-array (FPGA) featuring analog to digital (ADC) and digital to analog (DAC) converters. The signal from a position sensitive photodiode (PD) is amplified (INA111) and low-pass anti-alias filtered (AAF). Digital outputs control digital direct synthesizers (DDS) which drive acousto-optic deflectors (AOD). 


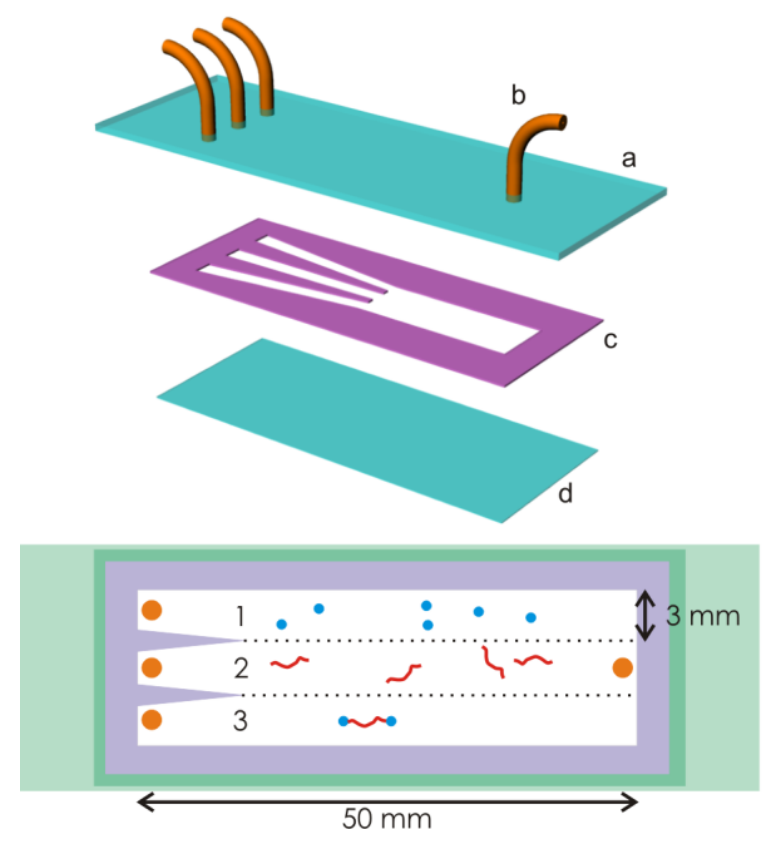

FIG. 4. (Color online) A sample chamber (top) was assembled by drilling $1.6 \mathrm{~mm}$ holes in a $75 \times 25 \times 1 \mathrm{~mm}^{3}$ microscope slide (a) and by glueing $0.25 \mathrm{~mm}$ i.d. PEEK tubing (b) to the slide using UV-curing epoxy (Norland NOA81). A 3-lane pattern was then cut into a $200 \mu \mathrm{m}$ thick doublestick tape spacer (c, Tesa) which was glued to the slide. The chamber was sealed with a $60 \times 24 \times 0.17 \mathrm{~mm}^{3}$ coverslip (d, Corning). (bottom) Experiments were performed by trapping beads in channel 1, finding a DNA-tether in channel 2, and performing force-extension and force-clamp experiments in channel 3. 


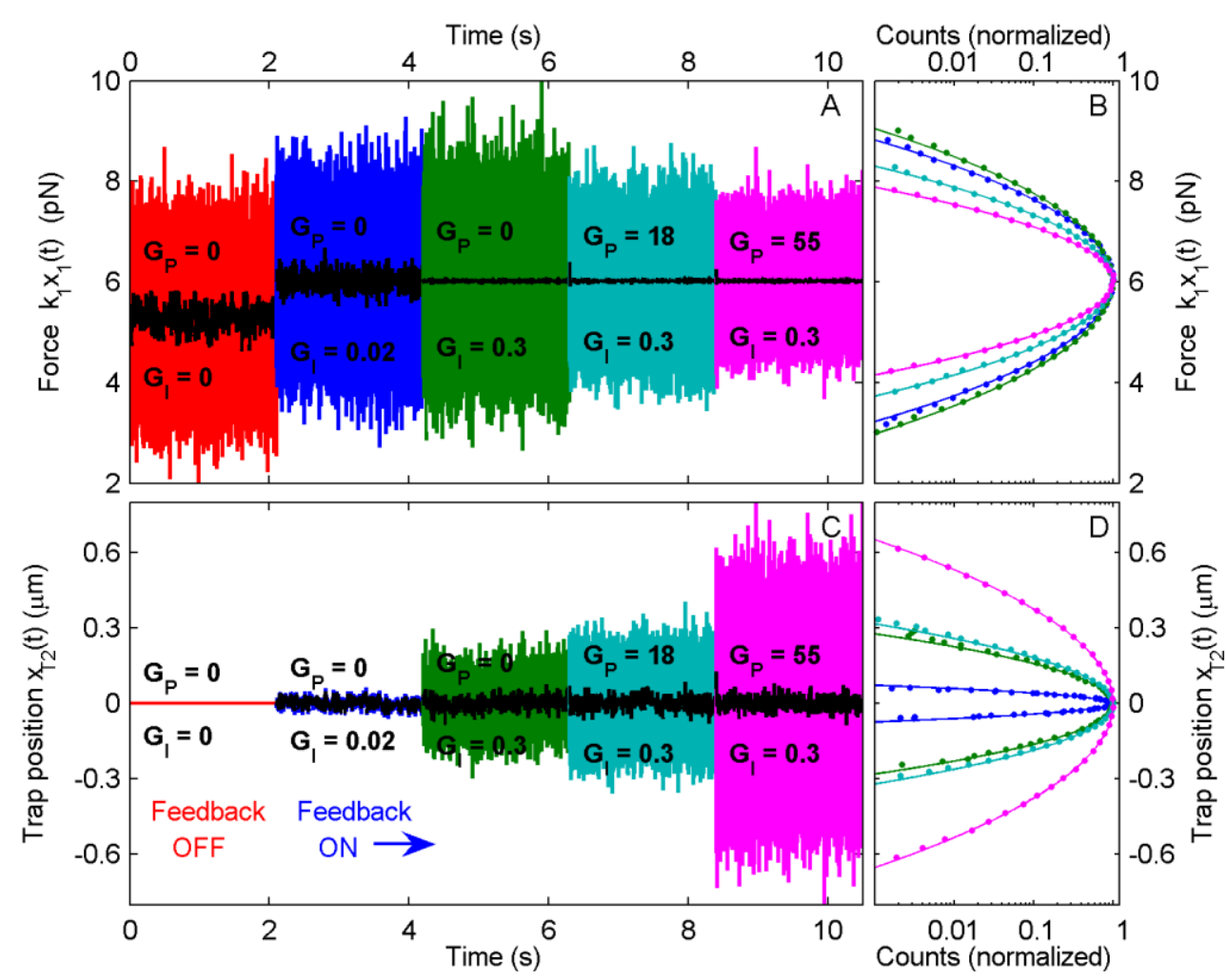

FIG. 5. (Color online) (A) Time-series of force-signal, collected at $200 \mathrm{kS} / \mathrm{s}$, during a force-clamp experiment. The set-point force was $6 \mathrm{pN}$. The proportional gain $G_{P}$ and integral gain $G_{I}$ were adjusted every $2 \mathrm{~s}$. (B) Histograms of the force remain gaussian, which indicates harmonic trapping. (C) Time-series of trap position $x_{T 2}$, collected at $200 \mathrm{kS} / \mathrm{s}$, during the force-clamp experiment, and its corresponding histogram (D). The black traces show data low-pass filtered to $100 \mathrm{~Hz}$. 

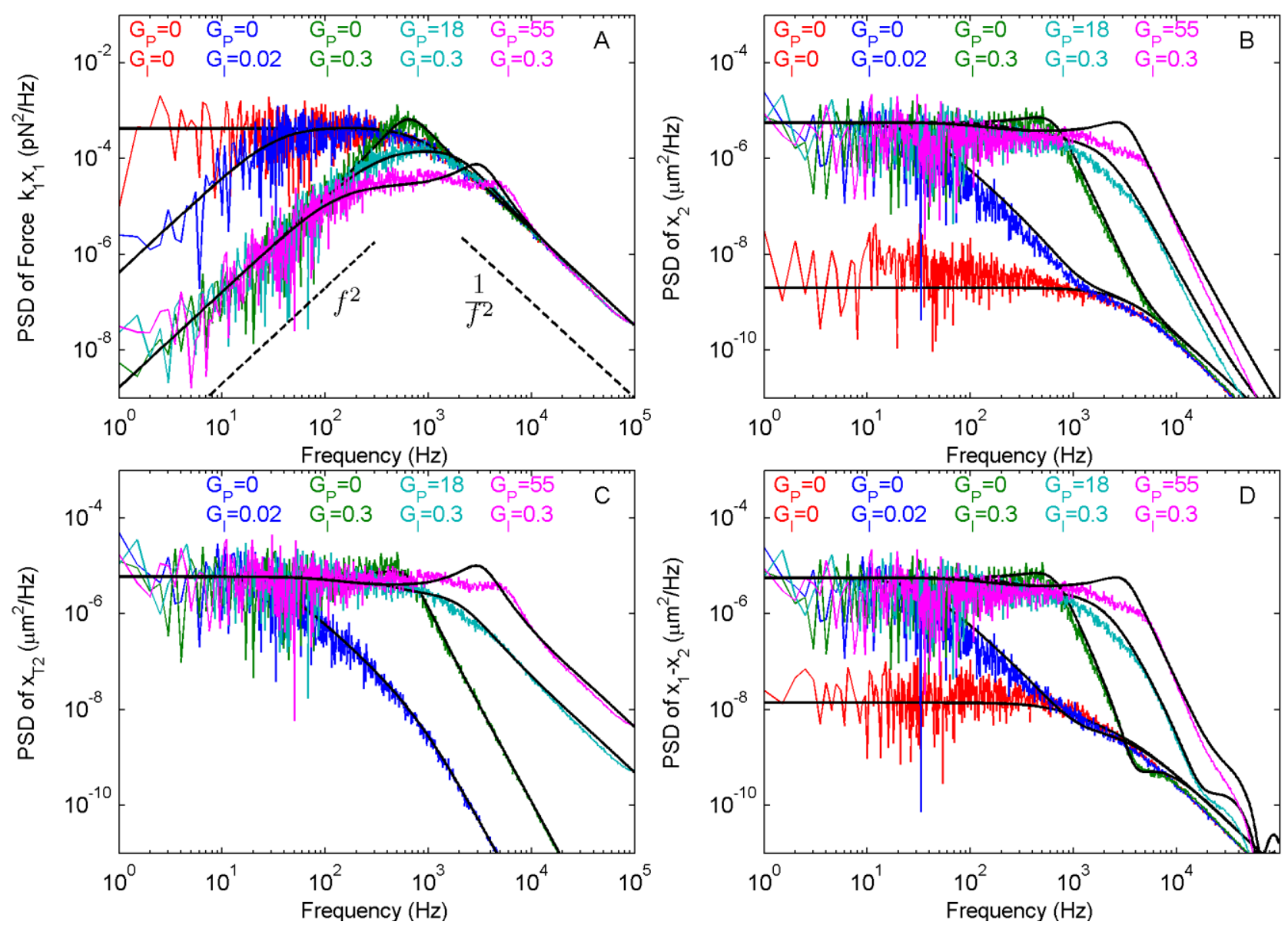

FIG. 6. (Color online) Power spectral densities of force (A), steerable bead position (B), trap position (C), and tether extension (D). The predicted PSD (Eq.7) is shown in black and (A) is shown with dashed lines proportional to $f^{2}$ and $f^{-2}$ as a guide to the eye. 


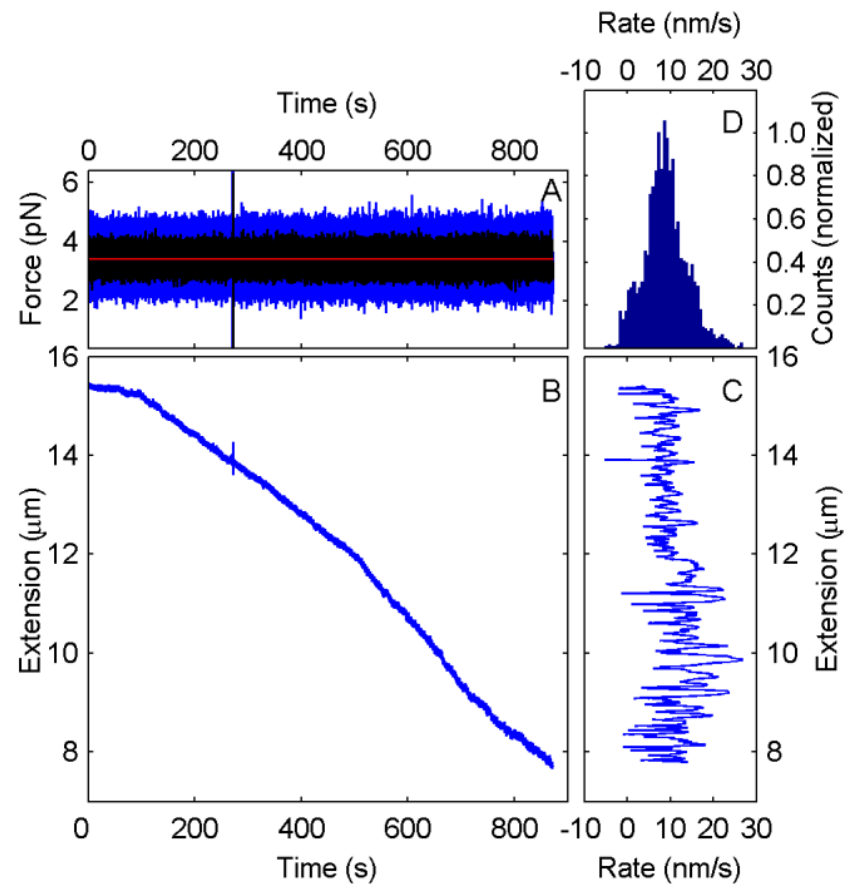

FIG. 7. (Color online) (A) Measured force and (B) tether extension during the enzymatic activity of lambda exonuclease. The red line in (A) shows the force set-point, $3.4 \mathrm{pN}$, for the force clamp control. The blue trace shows the force signal at $2 \mathrm{kHz}$ bandwidth, while the black trace shows data low-pass filtered to $100 \mathrm{~Hz}$. (C) Rate of the extension change calculated from time-series in (B) (see text). (D) Histogram of the rates showing an average rate of $9 \pm 6 \mathrm{~nm} / \mathrm{s}$. 
$t=805 s$

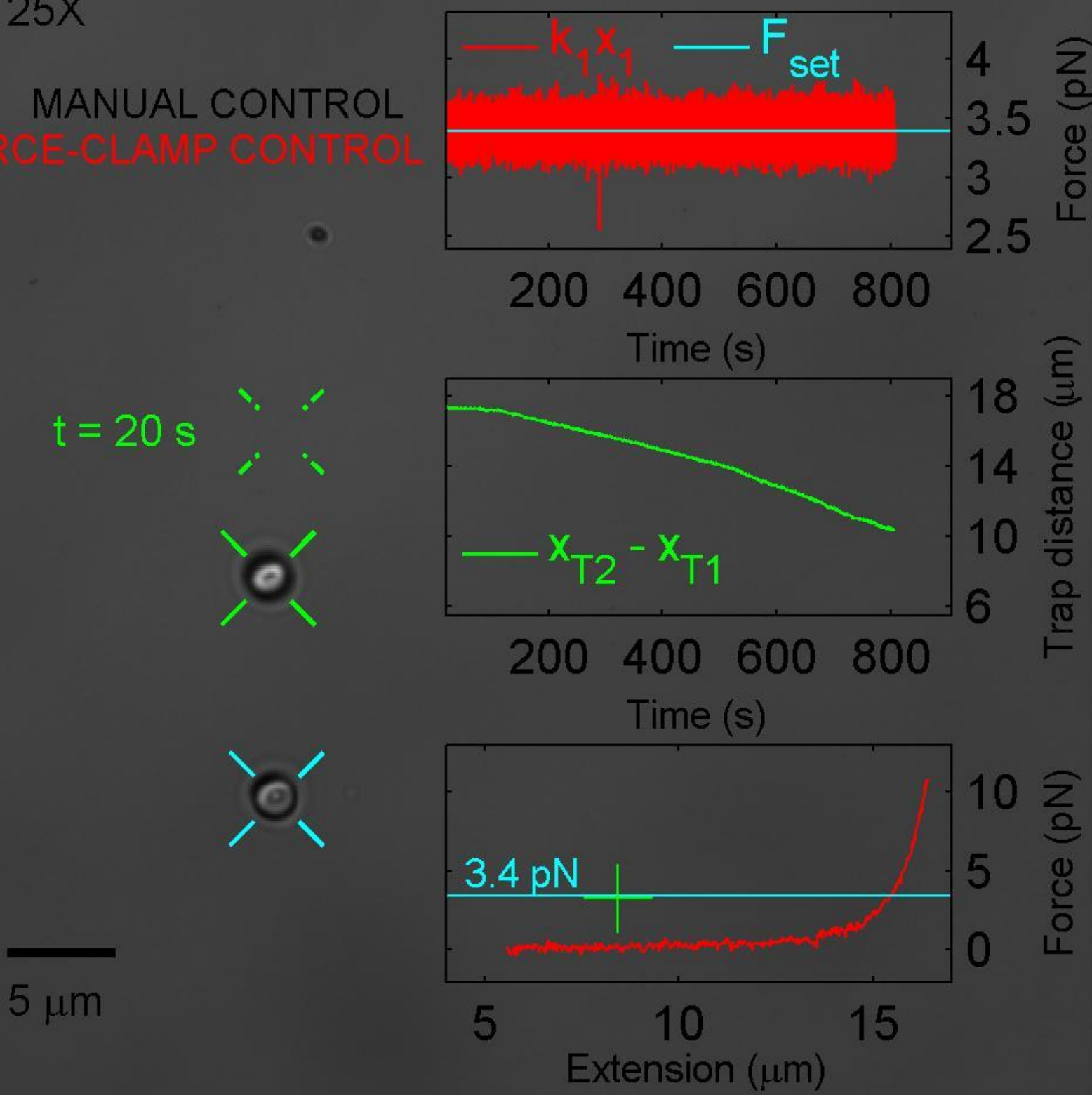

FIG. 8. Single-frame excerpt from video recording of force clamp experiment with lamda exonuclease. In the first part of the video a force-extension curve (bottom panel) is obtained using manual control. In the second part, after $\mathrm{t}=20 \mathrm{~s}$, the tether is held force clamped at $3.4 \mathrm{pN}$ (force shown in top panel). The video is at normal speed $(1 \mathrm{x})$ while the force extension curve is measured. During 13 min of force-clamp control the video is sped up 25-fold. The gradual conversion from a double-stranded tether to a single-stranded tether is seen as a decrease in the extension (middle panel). The tether broke at $\mathrm{t}=880 \mathrm{~s}$. Scale-bar $5 \mu \mathrm{m}$. (enhanced online). 